\title{
The scent fingerprint of hepatocarcinoma: in-vitro metastasis prediction with volatile organic compounds (VOCs)
}

This article was published in the following Dove Press journal:

International Journal of Nanomedicine

27 July 2012

Number of times this article has been viewed

\author{
Haitham Amal ${ }^{1, \neq}$ \\ Lu Ding ${ }^{2, \ddagger}$ \\ Bin-bin Liu ${ }^{3, \neq}$ \\ Ulrike Tisch' \\ Zhen-qin $\mathrm{Xu}^{2}$ \\ Da-you Shi ${ }^{2}$ \\ Yan Zhao ${ }^{3}$ \\ Jie Chen ${ }^{3}$ \\ Rui-xia Sun ${ }^{3}$ \\ $\mathrm{Hu}$ Liu $^{2}$ \\ Sheng-Long $\mathrm{Ye}^{3}$ \\ Zhao-you Tang ${ }^{3}$ \\ Hossam Haick'
}

'Department of Chemical Engineering and Russell Berrie Nanotechnology Institute, Technion - Israel Institute of Technology, Haifa, Israel; ${ }^{2}$ Department of Oncology, The First Affiliated Hospital of Anhui Medical University, Hefei, China; ${ }^{\text {LLiver Cancer }}$ Institute and Zhong-shan Hospital, Fudan University, Shanghai, China

¥These authors have equal contribution to the manuscript

Correspondence: Hossam Haick

The Department of Chemical Engineering and Russell Berrie Nanotechnology Institute,

Technion - Israel Institute of Technology, Haifa 32000, Israel

Tel +972 48293087

Fax +972 48295672

Email hhossam@technion.ac.il

Hu Liu

Department of Oncology, the First Affiliated Hospital of Anhui Medical University, Hefei 230022, China.

Fax +86 55I 2922987

Email drliuhu@gmail.com

Sheng-Long Ye

Liver Cancer Institute \& Zhongshan Hospital,

Fudan University, Shanghai, 200032, China.

Fax +86 2I 6403 7I8I

Email slye@shmu.edu.cn
Background: Hepatocellular carcinoma (HCC) is a common and aggressive form of cancer. Due to a high rate of postoperative recurrence, the prognosis for HCC is poor. Subclinical metastasis is the major cause of tumor recurrence and patient mortality. Currently, there is no reliable prognostic method of invasion.

Aim: To investigate the feasibility of fingerprints of volatile organic compounds (VOCs) for the in-vitro prediction of metastasis.

Methods: Headspace gases were collected from 36 cell cultures (HCC with high and low metastatic potential and normal cells) and analyzed using nanomaterial-based sensors. Predictive models were built by employing discriminant factor analysis pattern recognition, and the classification success was determined using leave-one-out cross-validation. The chemical composition of each headspace sample was studied using gas chromatography coupled with mass spectrometry (GC-MS).

Results: Excellent discrimination was achieved using the nanomaterial-based sensors between (i) all HCC and normal controls; (ii) low metastatic HCC and normal controls; (iii) high metastatic HCC and normal controls; and (iv) high and low HCC. Several HCC-related VOCs that could be associated with biochemical cellular processes were identified through GC-MS analysis.

Conclusion: The presented results constitute a proof-of-concept for the in-vitro prediction of the metastatic potential of HCC from VOC fingerprints using nanotechnology. Further studies on a larger number of more diverse cell cultures are needed to evaluate the robustness of the VOC patterns. These findings could benefit the development of a fast and potentially inexpensive laboratory test for subclinical HCC metastasis.

Keywords: hepatocarcinoma, metastasis, volatile organic compound, sensor, GC-MS

\section{Introduction}

Hepatocellular carcinoma (HCC) is a particularly aggressive cancer with a very dismal prognosis and is especially prevalent in Asia and Africa. ${ }^{1-3}$ Curative surgical resection is the preferred treatment for HCC. Early detection of liver cancer and advances in surgical techniques have greatly improved short term survival for those diagnosed with HCC. ${ }^{4}$ However, at 5 years, the rate of postoperative recurrence is as high as $70 \%$, which poses a major obstacle for a patient's long-term survival. ${ }^{4-6}$ Subclinical metastases from the original tumor that occurred before surgery are the major causes of tumor recurrence and patient mortality from liver cancer., ${ }^{4,5}$ Most HCCs reoccur near the primary lesion soon after surgery; hence, large-scale liver resection and adjuvant chemotherapy would, in principle, be advantageous to the patient. However, severe cirrhosis in HCC patients often makes large-scale hepatic resection extremely risky. ${ }^{4}$ Chemotherapeutic drugs 
have a wide range of adverse side-effects and associated comorbidities; one of these side-effects is hepatotoxicity, which poses a particular risk to HCC patients. In laboratory settings, the metastatic potential of malignant cells is best assayed in vivo by inoculation of the malignant cells into nude mice, followed by counting the number of metastatic foci in the lung. ${ }^{7-10}$ The metastatic potential can also be assayed by a variety of widely used cell-level techniques, such as cell adhesion, migration, invasion, and angiogenesis. ${ }^{7}$ However, these methods are time-consuming, costly, and unsuitable for clinical application. There is no suitable method that is currently being used in clinical practice to reliably predict the therapeutic outcome of liver resection. Hence, a new rapid, cost-effective, and accurate prognostic method of invasion is needed.

The analysis of volatile organic compounds (VOCs) is emerging as a new frontier in medical diagnostics for a variety of diseases; among these diseases are cancers of the lung, breast, colon, prostate, and head-and-neck, as well as kidney disease and neurodegenerative diseases. ${ }^{11-25}$ This approach is based on the emission of VOCs from the cell membranes that primarily consist of amphipathic phospholipids, carbohydrates, and many integral membrane proteins. The cell membranes of different cell types are distinct. ${ }^{26,27}$ Tumor growth and metastasis formation is accompanied by gene and/or protein changes, which lead to oxidative stress and peroxidation of the cell membrane species. This, in turn, causes the emanation of a characteristic mixture of VOCs. ${ }^{28-30}$ Depending on whether a cell is normal or malignant, some of these VOCs appear in distinctively different mixture compositions. ${ }^{11,13,22,23}$ These VOCs can be detected directly from the headspace of the cells.

It has recently been shown that arrays of cross-reactive nanomaterial-based sensors can identify and separate several types of lung cancer with different histologies, based on the analysis of in-vitro headspace samples from cell lines, ${ }^{13}$ using pattern recognition methods. Liver cancer-related
VOCs have been identified in exhaled breath, ${ }^{31}$ as well as in blood. ${ }^{32}$ In this pilot study, we have adapted the nanomaterialsensor-technology for in-vitro differentiation between subtle differences in the VOC profiles of HCC cells with different potentials for metastasis formation. We have demonstrated the ability of the sensors to discriminate between HCC with normal cells, as well as between HCC cells with high and low metastatic potential. Complementary chemical analysis of the headspace samples identified several headspace VOCs that are able to distinguish between the studied cell types, which allows for the tracking of the different cells' metabolic pathways.

\section{Materials and methods Cell cultures and headspace sample collection}

Six cell lines (MHCC97-H; MHCC97-L; HepG2; SMMC7721; BEL-7402; L-02) were obtained from the Liver Cancer Institute, Zhongshan Hospital (Shanghai, China). ${ }^{8,9,33-37}$ MHCC97-H was used as a model for HCC cells with high metastatic potential (HCC-HMP); ${ }^{8,9,33,37}$ MHCC97-L, HepG2, SMMC-7721, and BEL-7402 were used as models for HCC cells with low metastatic potential (HCC-LMP). ${ }^{8,9,33-37}$ The cell line model was obtained through in vivo clonal selection. ${ }^{9}$ Table 1 lists the rate of spontaneous pulmonary metastasis of recipient nude mice after inoculation (HCC-HMP: 100\%; HCC-LMP: $<50 \%$ ). All cell line replicas were validated less than 6 months prior to the experiment to ensure that they had not undergone additional mutations that would cause them to no longer reflect the properties (including the metastatic potential) of the cell they were derived from. In this study, we cultured the cell lines under the conditions suggested by the supplier in order to preserve their characteristics after in-vitro passages. ${ }^{38}$

Comparative analysis of cell lines that differ in their metastatic potential could, in principle, lead to a distinction

Table I Cell culture characteristics and spontaneous pulmonary metastasis of recipient nude mice after inoculation

\begin{tabular}{lllll}
\hline Category & Cell line & $\begin{array}{l}\text { Rate of spontaneous } \\
\text { pulmonary metastasis }\end{array}$ & Reference & Growth medium \\
\hline HCC-HMP & MHCC97-H & $100 \%(10 / 10)$ & $8,9,33,37$ & DMEM with I0\% FBS \\
HCC-LMP & MHCC97-L & $40 \%(4 / 10)$ & $8,9,33,37$ & \\
& SMMC772I & $33 \%(2 / 6)$ & 34 & \\
& HepG2 & $0 \%(0 / 10)$ & 35 & \\
Normal cells & BEL-7402 & $10.4 \%(5 / 48)$ & & \\
\hline
\end{tabular}

Notes: All cell lines were sub-cultured for six repetitions, and two separate headspace samples were collected from each cell line repetition for GC-MS analysis and analysis with the sensors array.

Abbreviations: GC-MS, gas chromatography coupled with mass spectrometry; HCC-HMP, hepatocellular carcinoma cells with high metastatic potential; HCC-LMP, hepatocellular carcinoma cells with low metastatic potential; DMEM, Dulbecco's modified eagle medium; FBS, fetal bovine serum. 
due to an unrelated property. Using multiple HCC-LMP cell lines reduces this possibility by simulating the natural diversity of cancer cells. In this research, the human immortalized hepatocyte cell line L-02 served as a model for normal cell activity. All cell lines were cultured in complete media (Dulbecco's modified eagle medium [DMEM] with 10\% fetal bovine serum [FBS]) according to the manufacturer's instruction. Cell growth was conducted in $25 \mathrm{~cm}^{2}$ flasks (Nunclon) at $37^{\circ} \mathrm{C}$ in a $5 \% \mathrm{CO}_{2}$ incubator. At $90 \%$ confluence, the cells were sub-cultured for six repetitions. A medium for the same incubation time and conditions, but without cells, served as a background control. The headspace samples were collected (in duplicates for GC-MS analysis and analysis with the sensors array) at $90 \%$ confluence from seeding, by connecting two parallel two-bed ORBO ${ }^{\mathrm{TM}} 420$ Tenax $^{\circledR}$ TA sorption tubes for gas and vapor sampling (specially treated; 35/60 mesh; 100/50 mg; purchased from Sigma-Aldrich, China). Highly pure nitrogen $(5 \mathrm{~N})$ was flown as carrier gas through the flask for $10 \mathrm{~min}$ at a constant rate of $50 \mathrm{~mL} / \mathrm{min}$ to transfer the sample to the sorbent tubes. The maximal duration between sample collection and analysis was 2 months. In a separate experiment, we verified that the samples can be stored in the ORBO ${ }^{\mathrm{TM}} 420 \mathrm{Tenax}^{\circledR}$ TA sorption tubes for at least 6 months.

\section{Study design}

The headspace analysis was comprised of the following four comparative studies: (i) all HCC cell cultures versus the normal control cell cultures; (ii) the HCC cell cultures with low metastatic potential (ie, spontaneous pulmonary metastasis in $<50 \%$ of nude mice) versus the normal controls; (iii) the HCC cell cultures with high metastatic potential (ie, spontaneous pulmonary metastasis in $100 \%$ of nude mice) versus the normal controls; and (iv) the HCC cell cultures with high metastatic potential versus the ones with low metastatic potential.

As described above, the samples were collected in duplicates from each cell line replica and were analyzed using two independent approaches: (i) chemical analysis with the aim to identify the headspace VOCs that show statistically different concentrations between the study groups, using gas-chromatography/mass-spectrometry (GC-MS; see GC-MS analysis of the headspace samples and GC-MS sections); (ii) analysis with an array of nanomaterial-based sensors, combined with a statistical pattern recognition algorithm (see Headspace analysis with the nanomaterial-based sensors and Sensor Array sections), with the aim of identifying specific patterns of HCC cells and metastatic potential.

\section{GC-MS analysis of the headspace samples}

The headspace VOCs were identified using a GCMSQP2010 instrument (Shimadzu Corporation, Japan) with a SLB-5 ms capillary column (with 5\% phenyl methyl siloxane; $30 \mathrm{~m}$ length; $0.25 \mathrm{~mm}$ internal diameter; $0.5 \mu \mathrm{m}$ thicknesses; from Sigma-Aldrich), combined with a thermal desorption (TD) system (TD20; Shimadzu Corporation, Japan). The oven temperature profile was set as follows: (i) $35^{\circ} \mathrm{C}$, $10 \mathrm{~min}$; (ii) $4^{\circ} \mathrm{C} / \mathrm{min}$ ramp until $150^{\circ} \mathrm{C}$, (iii) $10^{\circ} \mathrm{C} / \mathrm{min}$ ramp until $300^{\circ} \mathrm{C}$; (iv) $300^{\circ} \mathrm{C}, 15 \mathrm{~min}$. Prior to analysis, the Tenax material from the $\mathrm{ORBO}^{\mathrm{TM}} 420 \mathrm{Tenax}^{\circledR}$ TA sorption tubes (one of the two tubes per cell line replica) that had absorbed the headspace VOCs was transferred to pre-cleaned and preconditioned empty glass TD tubes (from Sigma-Aldrich) that were compatible with the TD system. The transfer was carried out in a glove box in a dry $\mathrm{N}_{2}$ atmosphere and at constant temperature $\left(\mathrm{T}=21^{\circ} \mathrm{C}\right)$. The filled TD tubes were injected into the GC-system in splitless mode at $30 \mathrm{~cm} / \mathrm{sec}$ constant linear speed and under $0.70 \mathrm{~mL} / \mathrm{min}$ column flow. The TD temperature was set to $250^{\circ} \mathrm{C}$. Contaminants of the Tenax sorbent material were identified through GC-MS analysis of pristine Tenax material from unused $\mathrm{ORBO}^{\mathrm{TM}} 420 \mathrm{Tenax}^{\circledR}$ TA sorption tubes.

The GC-MS chromatograms were analyzed using the GCMS post-run analysis program (GCMS solutions version 2.53SU1, Shimadzu Corporation), and the compounds were tentatively identified through spectral library match (Compounds library of the National Institute of Standards and Technology, Gaithersburg, MD 20899-1070 USA). The data were processed using the open source XCMS package version 1.22.1 for R environment (http://metlin.scripps.edu/ download/), which provides $\mathrm{m} / \mathrm{z}$ and retention times.

\section{Headspace analysis with the nanomaterial-based sensors}

In order to release the headspace VOCs, the Tenax sorbent material from the second $\mathrm{ORBO}^{\mathrm{TM}} 420$ Tenax $^{\circledR}$ TA sorption tube per patient was heated at $270^{\circ} \mathrm{C}$ for $10 \mathrm{~min}$ in a pre-heated $750 \mathrm{~mL}$ stainless steel TD chamber. Pulses of the sample from the TD chamber were then delivered by a gas sampling system into a stainless steel test chamber that contained an array of cross-reactive nanomaterial-based sensors, which are described below. The test chamber was evacuated between exposures to release the VOCs that the sensors adsorbed. An Agilent multifunction switch 34980 was used to simultaneously measure the resistance of the sensors as a function of time. The sensors' baseline responses were recorded for 5 min in vacuum, followed by 5 min under headspace sample 
exposure, followed by another $5 \mathrm{~min}$ in vacuum. During analyte exposure and/or during the relaxation period, one or two sensing features were read out from the time-dependent resistance response of each sensor that was related to the area underneath the time-dependent resistance response.

The nanomaterial-based sensor array that was used to analyze the headspace samples contained cross-reactive, chemically diverse chemiresistors that were based on two types of nanomaterials: (i) organically stabilized spherical gold nanoparticles (GNPs, core diameter: 3-4 nm) and (ii) single walled carbon nanotubes (SWCNTs) (see Table 2). The chemical diversity of the sensors was achieved through eleven different organic functionalities (seven for the GNP sensors and four for the SWCNT sensors) that are listed in Table 2.

The GNPs were synthesized as described in previous publications ${ }^{11,20}$ and were dispersed in chloroform. Chemiresistive layers with an electrical resistance of several $\mathrm{M} \Omega$ were formed by drop-casting the solution onto semi-circular microelectronic transducers. The device was dried for $2 \mathrm{~h}$ at ambient temperature and then baked overnight at $50^{\circ} \mathrm{C}$ in a vacuum oven. The microelectronic transducers consisted of ten pairs of circular interdigitated (ID) gold electrodes on silicon with $300 \mathrm{~nm}$ thermal oxide (Silicon Quest International, Nevada, US) (see Supplementary Figure S1; available online). The outer diameter of the circular electrode area was $3 \mathrm{~mm}$, and the gap between two adjacent electrodes and the width of each electrode was 20 microns. The chemiresistive GNP layers connected the electrodes and formed multiple conductive paths between them. In these films, the metallic cores provided the electric conductivity and the organic ligands of the GNPs provided cross-selective absorption sites for the headspace VOCs. ${ }^{11,20,39}$ In addition to their role as an adsorptive phase, the presence of well-defined organic spacers (ie, capping molecules) aided in controlling the inter-particle distance, and thereby, the inter-particle distances in the composite films. This, in turn, resulted in low signal-to-noise ratios.

The SWCNT sensors were based on electrically continuous random networks of SWCNTs that were formed by dropcasting a solution of SWCNTs (from ARRY International LTD, Germany; $\sim 30 \%$ metallic, $\sim 70 \%$ semiconducting, average diameter $=1.5 \mathrm{~nm}$, length $=7 \mathrm{~nm}$ ) in dimethylformamide (DMF, from Sigma Aldrich Ltd, $>98 \%$ purity) onto the prepared electrical transducers. After deposition, the devices were slowly dried overnight under ambient conditions to enhance the self-assembly of the SWCNTs and to evaporate the solvent. The procedure was repeated until a resistance of $100 \mathrm{~K} \Omega$ to $10 \mathrm{M} \Omega$ was obtained. The microelectronic transducers for the SWCNT sensors consisted of ten pairs of $4.5 \mathrm{~mm}$ wide, interdigitated platinum electrodes on silicon with two microns of thermal oxide (Silicon Quest International, Nevada, US). The gap between the two adjacent electrodes was 100 microns wide. The SWCNT sensors were organically functionalized with cap-layers that were composed of one Hexabenzocoronene (HBC) derivative and three Polycyclic Aromatic Hydrocarbon (PAH) derivatives. ${ }^{40-42}$

The GNP and SWCNT/PAH or SWCNT/HBC sensors used in this study responded rapidly and reversibly when exposed to typical headspace VOCs..$^{40,41,43}$ Additionally, we have confirmed that they have a very low response to water. ${ }^{11,40-42,44}$ This is an important feature, because, otherwise, a

Table 2 The organic functionalities of the nanomaterial-based sensors

\begin{tabular}{|c|c|c|c|c|c|}
\hline \multirow[t]{2}{*}{ Base material } & \multirow[t]{2}{*}{ Organic functionality } & \multicolumn{4}{|l|}{ DFA model no } \\
\hline & & $\mathbf{I}$ & 2 & 3 & 4 \\
\hline & & Test group & & & \\
\hline & & $\mathrm{HCC}$ & HCC-LMP & HCC-HMP & HCC-HMP \\
\hline & & Control group & & & \\
\hline & & Normal cells & Normal cells & Normal cells & HCC-LMP \\
\hline \multirow[t]{7}{*}{ GNPs } & Decanethiol & $x$ & & & \\
\hline & Octadecanethiol & & & & $x$ \\
\hline & Tert-dodecanethiol & $x$ & & & \\
\hline & 2-Mercaptobenzimidazole & & & & $x$ \\
\hline & 2-Naphthalenethiol & & $x$ & & $x$ \\
\hline & 2-Ethylhexyl-3-mercaptopropionate & $x$ & $x$ & & \\
\hline & Benzylmercaptan & & & $x$ & \\
\hline \multirow[t]{4}{*}{ SWCNTs } & $\mathrm{HBC}-\mathrm{C}_{6,2}$ & $x$ & & & \\
\hline & $\mathrm{PAH}-2$ & & $x$ & & \\
\hline & $\mathrm{PAH}-5$ & & & $x$ & \\
\hline & $\mathrm{PAH}-7$ & $x$ & & & \\
\hline
\end{tabular}

Abbreviations: GNPs, gold nanoparticles; SWCNTs, single walled carbon nanotubes; PAH, polycyclic aromatic hydrocarbon; HBC, hexabenzocoronene; HCC, hepatocellular carcinoma; HCC-LMP, hepatocellular carcinoma cells with low metastatic potential; HCC-HMP, hepatocellular carcinoma cells with high metastatic potential. 
sensor's response to the high background humidity in the headspace could easily mask the signal to much lower concentrations of the headspace VOCs, which indicate disease.

\section{Statistical analysis GC-MS}

Shapiro-Wilk tests were employed to test and reject the null hypothesis for normal distribution of the GC-MS data within the study groups. The VOCs showing significant differences between the study groups were determined from the GC-MS results, using the non-parametric Wilcoxon/Kruskal-Wallis test for populations whose data cannot be assumed to be normally distributed. ${ }^{45,46}$ In this study, no correction for multiple testing was employed. All statistical analysis of the GC-MS data was carried out using JMP, Version 9.0.0 (SAS Institute Inc, Cary, NC, USA, 1989-2005).

\section{Sensor array}

Each sensor responded to all (or to a certain subset) of the headspace VOCs. Specific VOC patterns and predictive models for the studied cell types were derived from the sensor array output by applying discriminant factor analysis (DFA) as statistical pattern recognition algorithm for binary comparisons between the study groups. ${ }^{47}$ DFA is a linear, supervised pattern recognition method. The classes to be discriminated were defined before the analysis was performed. The DFA input variables were the features that were extracted from the sensors' responses (see section Headspace analysis with the nanomaterial-based sensors). The DFA algorithm determined the linear combinations of the input variables in such a way that the variance within each class was minimized and the variance between classes was maximized. By filtering out non-contributing sensors, DFA also served as a heuristic to select the sensing features that significantly contributed to the separation between the study groups. The reason for selecting a certain set of sensing features for a particular problem was based on their ability to discriminate between the various classification groups. The DFA output variables (ie, canonical variables) were obtained in mutually orthogonal dimensions; the first canonical variable, CV1, was the most powerful discriminating dimension. Thus, DFA effectively reduced the multidimensional experimental data. The clusters were distinguished through statistical treatment of the CV1 values.

The classification success rate of the binary comparisons was estimated through leave-one-out cross validation. For this purpose, DFA was computed using a training data set that excluded one test sample. After the DFA computation, the test sample was projected onto the CV1 axis that was calculated using the training set. Thereby, the test sample was "blinded" against the DFA model, so that its class affiliation was unknown. All possibilities of leaving out one sample were tested and the left out samples were classified as true positive (TP), true negative (TN), false positive (FP) and false negative (FN). Thus, given $n$ measurements, the DFA model was computed $n$ times using n-1 input vectors. The classification accuracy was estimated as the averaged performance over the $n$ tests.

All statistical analysis was performed using JMP, Version 9.0.0 (SAS Institute Inc, Cary, NC, USA, 1989-2005).

\section{Results \\ Chemical analysis of the headspace cell lines}

Our GC-MS analysis identified over 500 different VOCs in each headspace sample. GC-MS analysis of pristine Tenax material from unused ORBO ${ }^{\mathrm{TM}} 420$ Tenax $^{\circledR}$ TA sorption tubes identified methylene chloride, acetaldehyde, L-cysteine sulfonic acid, malonic acid, and naphthalene as possible contaminants of the Tenax sorbent material. These substances were disregarded in the subsequent comparative analysis. The VOCs with over $0.02 \%$ of the total amount detected by GC-MS (typically 300 per headspace sample) were further analyzed. We first tracked the 179 VOC that were present in $>80 \%$ of the samples. Shapiro-Wilk tests showed that the null hypothesis for normal distribution of the GC-MS data was not fulfilled for these VOCs. Therefore, non-parametric Wilcoxon/Kruskal-Wallis tests with a cut-off value of $P=0.05$ were used for the comparative analysis of the GC-MS data, which identified a total of nine VOCs that were, on average, significantly elevated or reduced between the compared groups (see Table 3 ). When compared to the normal cell cultures, only one compound (methanesulfonil chloride) was, on average, elevated in all HCC cells, irrespective of their metastatic potential. When separately comparing the two HCC cell subtypes (viz high and low metastatic potential) to the normal control cells, we identified three and one distinguishing VOCs that were either significantly elevated or reduced (see Table 3 ). Furthermore, the level of 2,3 di-hydro-benzofuran differentiated very clearly between HCC-LMP and HCC-HMP (see Table 3).

\section{Identification of patterns for HCC and its metastatic potential using nanomaterial- based sensors}

The sensor-based pattern recognition of HCC and its metastatic potential was based on the observed subtle differences 
Table 3 Headspace VOCs that showed significant statistical differences between the study groups

\begin{tabular}{|c|c|c|c|c|c|c|}
\hline \multirow[t]{2}{*}{ Retention time [min] } & \multirow[t]{2}{*}{$\mathrm{m} / \mathbf{z}$} & \multirow[t]{2}{*}{ Tentative name } & \multicolumn{4}{|l|}{ Test group } \\
\hline & & & $\mathrm{HCC}$ & HCC-LMP & HCC-HMP & HCC-HMP \\
\hline & & & Control group & & & \\
\hline & & & Normal cells & Normal cells & Normal cells & HCC-LMP \\
\hline & & & $P$-values' and trends ${ }^{2}$ & & & \\
\hline 3.109 & 79 & Methane-sulfonyl chloride & $0.05(+)$ & & & \\
\hline 35.117 & 120 & 2,3 Di-hydro-benzofuran & & $0.028(-)$ & $0.047(+)$ & $0.001(+)$ \\
\hline 2.550 & 26 & Ethanol & & & $0.047(-)$ & \\
\hline 3.650 & 45 & Acetic acid & & & $0.047(+)$ & \\
\hline
\end{tabular}

Notes: 'Calculated using the Wilcoxon non-parametric method. ${ }^{2}(+)$ : Increased in the test group, as compared to the control group; $(-)$ : decreased in the test group, as compared to the control group.

Abbreviations: HCC, hepatocellular carcinoma; HCC-LMP, hepatocellular carcinoma cells with low metastatic potential; HCC-HMP, hepatocellular carcinoma cells with high metastatic potential; VOCs, volatile organic compounds.

in the concentration of the headspace VOCs. However, the concentrations of many other headspace VOCs remained unaffected. Some sensors in the array were especially sensitive to the classes of distinguishing VOCs, thus adding to the discrimination between the groups; others were more sensitive to the VOCs that showed no systematic changes, and, hence, added mainly noise. As a result, the choice of the sensors from which the input features for the DFA models were obtained was crucial for achieving well-defined patterns. However, no a priori knowledge of the distinguishing VOCs was necessary, because DFA was applied as a heuristic approach to identify the best sensor-subset for the different applications. During the analysis, we took care to keep the number of DFA input parameters low enough to avoid over-fitting.

The first DFA model was based on all collected headspace samples in order to demonstrate the feasibility of the nanomaterial-based sensors for identifying HCC (see Tables 1 and 4). Figure 1A shows the DFA plot obtained from the responses of five sensors with different organic functionalities (see Table 2). The $30 \mathrm{HCC}$ samples and the six normal controls formed two well-separated clusters without overlap along CV1. Leave-one-out cross-validation yielded excellent classification success with only one false negative classification (ie, one HCC cell culture was falsely classified as normal, see Table 4). The second DFA model was based on four sensors (see Table 2) and was able to fully distinguish the 24 HCC-LMP from the six normal controls along CV1 (see Figure 1B). Once again, excellent classification success was achieved through cross-validation, with only one false negative classification (see Table 4). Figure $1 \mathrm{C}$ shows the CV1 distribution of the third DFA model that was based on two sensors (see Table 2) and distinguished between the six HCC-HMP cell cultures with high metastatic potential and the six normal controls, with $100 \%$ classification success (see Table 4). The fourth DFA model (based on three sensors, see Table 2) distinguished, also without overlap, between 24 HCC-LMP and six HCC-HMP cultures (see Figure 1D). Table 4 lists, in terms of sensitivity, specificity, and accuracy, the classification success of the cross-validation procedure for all four DFA models.

Table 4 Statistical classification success, using DFA and cross validation.

\begin{tabular}{lllll}
\hline Statistical Parameter & Test group & & & HCC-HMP \\
& HCC & HCC-LMP & & HCC-HMP \\
& Control group & & Normal cells & HCC-LMP \\
\hline Accuracy (\%) & Normal cells & 96.7 & 100 & 96.7 \\
Sensitivity (\%) & 97.2 & 95.8 & 100 & 83.3 \\
Specificity (\%) & 96.7 & 100 & 100 & 100 \\
True Positives & 100 & 23 & 6 & 5 \\
True Negatives & 29 & 6 & 6 & 24 \\
False Positives & 6 & 0 & 0 & 0 \\
False Negatives & 0 & 1 & 0 & 1 \\
\hline
\end{tabular}



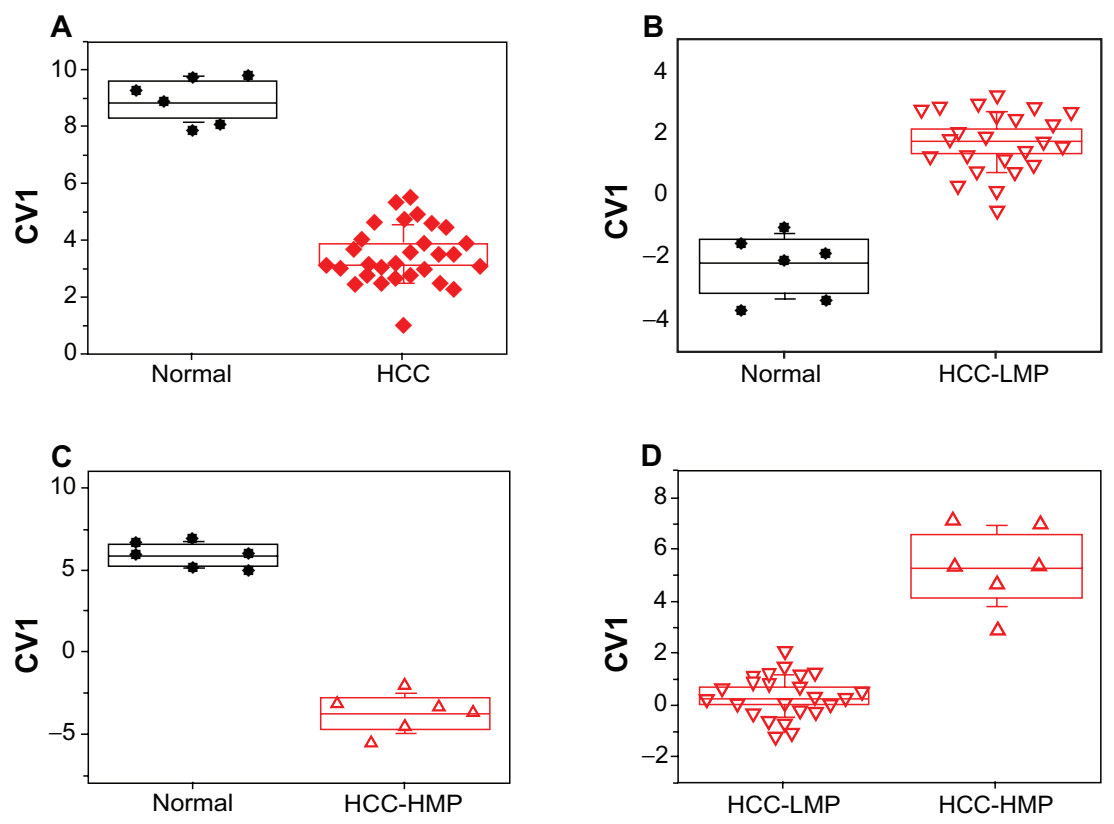

Figure I The DFA models that discriminated between (A) HCC cells (including cells with high and low metastatic potential) and normal control cells; (B) HCC-LMP cells and normal control cells; (C) HCC-HMP cells and normal control cells; and (D) HCC-LMP and HCC-HMP cells. Every point represents one cell culture.

Note: The sensors used for the four DFA models are listed in Table I.

Abbreviations: DFA, discriminant factor analysis; HCC, hepatocellular carcinoma; HCC-LMP, hepatocellular carcinoma cells with low metastatic potential; HCC-HMP, hepatocellular carcinoma cells with high metastatic potential.

\section{Discussion}

\section{In-vitro studies versus in-vivo and clinical studies}

This in-vitro pilot study has provided the first evidence of the existence of measurable VOC profiles of a tumor's metastatic potential. Studying the metabolic activity of isolated in-vitro cancer cells by analyzing their headspace VOCs avoids the organism's confounding factors (eg, variations in patient age, gender, lifestyle, medication, and other chronic diseases). Cell lines provide an abundant number of cells with similar characteristics, avoid variation between individuals, and bypass ethical issues associated with animal and human experiments. On the other hand, in-vitro studies may fail to replicate the precise cellular conditions, because they disregard the synergetic effect of cancer on the whole organism. Hence, these results, which are based on cell line studies, can only be considered as indicative, and their in-vivo translation will be far from trivial. Consequently, the translation to human samples that would provide useful data for clinical application might seriously differ from the cell lines data. Cancer specific VOCs can be excreted, for instance, through the exhaled breath via the respiratory system, which controls the gas exchange in the human body. ${ }^{11,17,22-25,48}$ These easily (in-vivo) accessible breath VOCs could be products of the metabolic activity of the tumor itself. They could also be by-products of bacteria and necrotic reactions caused by local inflammation in the microenvironment of the tumor, or they could be partially re-emitted environmental toxins that were previously adsorbed to the body. ${ }^{48}$ In addition, systemic breath VOCs could be produced or consumed because of cancer-related changes elsewhere in the body, affecting the blood chemistry, and eventually being excreted via the respiratory system. ${ }^{48}$

In particular, the metastatic potential of cancer cells is strongly related to the tumor's local environment and its interaction with the host. Cancer invasion and metastasis is the ultimate phenotype of complex interactions between the cancer cells and the host defense mechanism. The process of metastasis consists of selection and sequential steps that include angiogenesis, detachment, motility, invasion of the extracellular matrix, intravasation, circulation, adhesion, extravasation into the organ parenchyma, and growth. ${ }^{7,49}$ Hence, the potential for a tumor to have metastasis might not only be related to intrinsic factors of the tumor, but also to the capacity of the host to respond to and inhibit metastasis.

In this study, the cell lines' metastatic potential was defined by the rate of spontaneous pulmonary metastasis of recipient nude mice after inoculation: the HCC-HMP cell line caused lung metastasis in $100 \%$ of the inoculated animals, and the four HCC-LMP cell lines caused lung metastasis in less than $50 \%$ of nude mice. However, one should consider that a cell line's tumorigenicity in mice 
might not accurately predict metastasis formation in humans. Mouse models of human cancer pathogenesis have become a central part of research because laboratory mice provide the most experimentally accessible mammalian model - one that shares organ systems, systemic physiology, and genes with humans. ${ }^{50,51}$ While there are many parallels between mouse and human carcinogenesis, there are also important differences in tumor pathogenesis and metastasis formation at the cellular and molecular levels. ${ }^{50,51}$ Hence, there are certain inherent limitations in the mouse models. The detected VOC signatures of the metastatic potential of tumor cells must ultimately be verified through studies in humans, either through studying the headspace of resected tumor tissue from patients, or through exhaled breath analysis.

\section{VOC markers of HCC and its metastatic potential}

During the comparative studies, four distinguishing VOCs were identified (see Table 3). There were distinct VOC profiles for the three study groups. Some of the distinguishing VOCs may have logical clinical explanations, and the origins of others are not yet fully understood. Note that the VOC identification is tentative and requires confirmation through GC-MS measurements of calibration standards.

On average, methane-sulfonyl chloride was elevated in all HCC cell lines, as compared to normal cell cultures. Indeed, sulphur-containing compounds are responsible for the characteristic odor in the breath of cirrhotic patients. ${ }^{52}$ The concentration of sulphur-containing compounds in the blood and breath of healthy subjects is very low. Impairment of the liver function is associated with incomplete metabolism of methionine in the transamination pathway, leading to increased levels of mercaptanes. These are then easily oxidized to their respective sulphides ${ }^{53}$ and can be directly observed in the headspace of the HCC cells.

Interestingly, the headspace differences were more pronounced when comparing HCC-HMP to the normal control cells than when comparing HCC-LMP to the normal control cells (see Table 3). Apparently, the observed disparity in the headspace VOC profiles reflects discrepancies in the biochemical cellular processes of HCC cells with different metastatic potentials. Indeed, differential protein analysis of two of the hepatocellular carcinoma cell strains with different metastatic potentials that were used in this study (MHCC97$\mathrm{H}$ and MHCC97-L) showed alterations in the levels of several proteins that are associated with tumor metastasis. ${ }^{37}$

We observed that the level of ethanol in the headspace HCC-HMP cells was decreased when compared to normal cells, whereas the level of acetic acid was increased. The enzymes that are responsible for converting alcohols to aldehydes and aldehydes to carboxylic acid are alcohol dehydrogenases (ADH) and aldehyde dehydrogenases (ALDH), respectively. Overexpression of these enzymes would lead to the observed alteration in the VOC profiles. Indeed, researchers found that overexpression of ADH and ALDH is correlated with liver cancer stem cells that play an important role in the formation of local and distant metastasis. ${ }^{54}$ In contrast, the levels of ethanol and acetic acid were unaltered in the headspace of HCC-LMP, as compared to the normal cells.

2,3 dihydro-benzofuran levels were increased in HCCHMP, as compared to normal control cells, but were decreased in HCC-LMP (see Table 3). This trend was confirmed through the direct comparison of HCC-HMP and HCC-LMP, showing very clear distinction between the two sub-groups ( $P=0.001$, see Table 3 ). Benzofuran derivatives can be absorbed from the environment. Food and drug regulatory agencies permit the use of limited levels of benzofuran in food containers and packaging. Chronic gavage exposure to 2,3-benzofuran was found to increase the frequency of tumors in several organs in rats and mice, but there is no evidence for carcinogenicity in humans. ${ }^{55}$ Recently, benzofuran derivatives have been evaluated for their cytotoxic potential against human cell lines, among them SMMC-7721, which was used in this study. ${ }^{56}$ These derivatives are now attracting interest as potentially effective chemopreventive or chemotherapeutic agents against malignant T-cells. ${ }^{57}$ The role of this substance in the development of metastasis is not yet clear and needs further clarification.

\section{Patterns of HCC and its metastatic potential from nanomaterial-based sensors}

Dense, well-separated patterns of CV1 were obtained from all four comparative DFA models (see Figure 1). Cross-validation confirmed that the results were stable and reproducible with different sub-sets of the samples (see section sensor Array). All four models had absolute specificity without false positive misclassifications, and high sensitivity with one false negative misclassification at most. Figure 1 shows that the clusters were most dense and their separation was most pronounced for the comparison between HCC-HMP and normal cells (see Figure 1C). The clusters spread and their separation decreased in the following order: $\mathrm{HCC}$ versus normal controls (see Figure 1A), HCC-HMP versus HCC-LMP (see Figure 1D), and HCC-LMP versus normal cells (see Figure 1B). This trend could reflect the 
assumed increase in metabolic activity, which would result in enhanced generation or consumption of headspace VOCs, from normal cells to HCC-LMP to HCC-HMP. This trend was indeed reflected in the observed VOC profiles, which showed statistically significant differences between normal cells and cells with high metastatic potential in 2,3 di-hydrobenzofuran, ethanol, and acetic acid, whereas the normal cells differed from cells with low metastatic potential only in the level of 2,3 di-hydro-benzofuran (see Table 3). This discrepancy could be due to the fundamental differences between the two approaches. Note, however, that the sensors in this study were broadly cross-reactive and all sensors responded to (a large subset of) the headspace VOCs of interest. ${ }^{40,44,58}$ Hence, the signals to the VOC mixture in the headspace samples were additive, so that the overall signal of one sensor stemmed from a total $\sim$ ppm amount of VOCs..$^{40,44,58}$ Therefore, the sensors' responses would be less affected by noise than the detected (sub) ppb concentrations of the separate headspace VOCs in the GC-MS analysis, and could potentially yield more reliable results.

The presented DFA models could hold future potential for a fast, single-step laboratory test for the simultaneous determination of liver malignancy and its metastatic potential. Such a test could be used in combination with conventional diagnostic methods. In an envisioned future clinical setting, a tissue specimen could be taken during liver biopsy or resection. At which point, the headspace above the tissue sample could be collected and analyzed on-site using a portable device with the eight sensors that were employed to build the first and the fourth DFA models (see Table 2). The first DFA model would determine whether the tissue sample contains cancer cells. In case of a positive result, the DFA model 4 could be applied immediately in order to determine the probability of metastasis formation. Treatment decisions could be made swiftly after liver biopsy, or even on-line during surgery. In these two models, the negligible possibility for false positive results would avoid unnecessary and potentially risky medical procedures and, thus, help to reduce patient mortality. A negative test result should be treated as complementary information for the conventional diagnostic tests, due to the possibility of false negative results in realistic settings. Note, however, that the estimated classification success and patterns stemmed from a small data set in this pilot study. A larger study that includes more (and more diverse) cell cultures is currently underway to confirm the obtained results and will be published elsewhere. It is reasonable to expect that the clusters for the larger sample size would be less defined and some overlap could occur. In turn, cluster separation could be improved again by further refining the choice of the sensors. The iterative improvement of the sensor set while expanding the sample size would eventually yield an optimized method.

\section{Conclusion and possible future clinical relevance}

We have delivered a proof-of-concept for the in-vitro identification of the scent print of HCC and its metastatic potential using nanomaterial-based sensors. Complementary chemical analysis tentatively identified several headspace VOCs that could be associated with liver-cancer-related biochemical processes, or with the accumulation of environmental toxins. The HCC scent print could, in principle, be utilized to predict metastasis and recurrence in a future laboratory test. While this pilot study cannot yet offer generalized conclusions forecasting a reliable use in diagnostics, it demonstrates the future potential for a fast prognostic assessment of metastasis. A future VOC-based laboratory test could complement conventional diagnostics after liver biopsy or liver resection, thus facilitating swift treatment decisions and patient follow-up, while helping to avoid unnecessary medical intervention. We expect that this approach is also viable for other types of cancer.

Following this proof-of-concept, a larger study that includes a higher number of more diverse cell cultures is underway to confirm the robustness of the VOC patterns. In particular, the further study will include several different HCC cell lines with high metastatic potential to match the number of cell lines with low potential for metastasis, as well as mixed cultures of healthy and HCC cells, in order to mimic physiological relevance by excluding a possible masking effect of the normal cells on the VOC signatures.

\section{Acknowledgment}

The authors acknowledge Dr Yoav Broza, Ms Orna Barash, and Ms Meggie Hakim for their assistance and support.

\section{Conflict of interest}

None of the authors declare any conflict of interest.

\section{Funding source}

The research leading to these results was funded by the FP7's ERC grant under DIAG-CANCER (grant agreement no $256639 ; H H)$.

\section{References}

1. Parkin D, Bray F, Ferlay J, Pisani P. Estimating the world cancer burden: Globocan 2000. Int J Cancer. 2001;94(2):153-156. 
2. Parkin D. International variation. Oncogene. 2004;23(38): 6329-6340.

3. Hussain SA, Ferry DR, El-Gazzaz G, et al. Hepatocellular carcinoma. Ann Oncol. 2001;12(2):161-172.

4. Nagao T, Inoue S, Yoshimi F, et al. Postoperative recurrence of hepatocellular carcinoma. Ann Surg. 1990;211(1):28-33.

5. Nagao T, Inoue S, Goto S, et al. Hepatic resection for hepatocellularcarcinoma: clinical features and long-term prognosis. Ann Surg. 1987;205(1):33-40.

6. Feng YX, Wang T, Deng YZ, et al. Sorafenib suppresses postsurgical recurrence and metastasis of hepatocellular carcinoma in an orthotopic mouse model. Hepatology. 2011;53(2):483-492.

7. Welch DR. Technical considerations for studying cancer metastasis in vivo. Clin Exp Metastasis. 1997;15(3):272-306.

8. Tian J, Tang ZY, Ye SL, et al. New human hepatocellular carcinoma (HCC) cell line with highly metastatic potential (MHCC97) and its expressions of the factors associated with metastasis. $\mathrm{Br} J$ Cancer. 1999;81(5):814-821.

9. Li Y, Tian B, Yang J, et al. Stepwise metastatic human hepatocellular carcinoma cell model system with multiple metastatic potentials established through consecutive in vivo selection and studies on metastatic characteristics. J Cancer Res Clin Oncol. 2004;130(8): 460-468.

10. Li JD, Peng Y, Dai Y, Li B, Wei YG, Yan LN. Comparison of the metastatic characteristics of HCCLM3 cells and SMCC-7721 cells in nude mice model. Zhonghua Gan Zang Bing Za Zhi. 2010;18(1):19-22.

11. Peng G, Tisch U, Adams O, et al. Diagnosing lung cancer in exhaled breath using gold nanoparticles. Nature Nanotechnol. 2009; 4(10):669-673.

12. Chen X, Xu F, Wang Y, et al. A study of the volatile organic compounds exhaled by lung cancer cells in vitro for breath diagnosis. Cancer. 2007;110(4):835-844.

13. Barash O, Peled N, Hirsch FR, Haick H. Sniffing the unique "odor print" of non-small-cell lung cancer with gold nanoparticles. Small (Weinheim an der Bergstrasse, Germany). Nov 2009;5(22):2618-2624.

14. Wehinger A, Schmid A, Mechtcheriakov S, et al. Lung cancer detection by proton transfer reaction mass-spectrometric analysis of human breath gas. Int J Mass spectrom. 2007;265(1):49-59.

15. Preti G, Labows JN, Kostelc JG, Aldinger S, Daniele R. Analysis of lung air from patients with bronchogenic carcinoma and controls using gas chromatography-mass spectrometry. J Chromatogr. 1988; 432:1-11.

16. O’Neill HJ, Gordon SM, O'Neill MH, Gibbons RD, Szidon JP. A computerized classification technique for screening for the presence of breath biomarkers in lung cancer. Clin Chem. 1988;34(8): 1613-1618.

17. Tisch U, Haick H. Arrays of chemisensitive monolayer-capped metallic nanoparticles for diagnostic breath testing. Rev Chem Eng. 2011;26:171-179.

18. Shuster G, Gallimidi Z, Heyman-Reiss A, et al. Classification of breast cancer precursors through exhaled breath. Breast Cancer Res Treat. 2011;126:791-796.

19. Tisch U, Haick H. Nanomaterials for cross-reactive sensor arrays. MRS Bull. 2010;35:797.

20. Tisch U, Haick H. Sensors based on monolayer-capped metal nanoparticles. In: Korotcenkov G, editor. Chemical Sensors - Vol 2: Nanstructured Materials. Vol 2 (Nanstructured Materials). 2010:141-202.

21. Tisch U, AlufY, Ionescu R, et al. Detection of asymptomatic nigrostriatal Dopaminergic lesion in rats by exhaled air analysis using carbon nanotube sensors. ACS Chem Neurosci. 2012;3(3):161-166.

22. Hakim M, Billan S, Tisch U, et al. Diagnosis of head-and-neck cancer from exhaled breath. Br J Cancer. 2011;104(10):1649-1655.

23. Peng G, Hakim M, Broza YY, et al. Detection of lung, breast, colorectal, and prostate cancers from exhaled breath using a single array of nanosensors. Br J Cancer. 2010;103(4):542-551.

24. Horváth I, Lázár Z, Gyulai N, Kollai M, Losonczy G. Exhaled biomarkers in lung cancer. Eur Respir J. 2009;34(1):261-275.
25. Amann A, Spanel P, Smith D. Breath analysis: the approach towards clinical applications. Mini Reviews in Med Chem. 2007;7(2):115-129.

26. Alberts B, Johnson A, Lewis J. Molecular Biology of the Cell. 4th ed. New York: Garland Publishing; 2002.

27. Singer SJ, Nicolson GL. The fluid mosaic model of the structure of cell membranes. Science. 1972;175:720-731.

28. Kneepkens CM, Lepage G, Roy CC. The potential of the hydrocarbon breath test as a measure of lipid peroxidation. Free Radic Biol Med. 1994;17(2):127-160.

29. Vousden KH, Ryan KM. p53 and metabolism. Nat Rev Cancer. Oct 2009;9(10):691-700.

30. Okunieff P, Fenton B, Chen Y. Past, present, and future of oxygen in cancer research. Adv Exp Med Biol. 2005;566:213-222.

31. Qin T, Liu H, Song Q, Song G, Wang H, Pan Y. The screening of volatile markers for hepatocellular carcinoma. Cancer Epidemiol Biomarkers and Prev. 2010;19(9):2247-2253.

32. Xue R, Dong L, Zhang S, et al. Investigation of volatile biomarkers in liver cancer blood using solid-phase microextraction and gas chromatography/mass spectrometry. Rapid Commun Mass Spectrom. 2008;22(8):1181-1186.

33. Li Y, Tang ZY, Ye SL, et al. Establishment of cell clones with different metastatic potential from the metastatic hepatocellular carcinoma cell line MHCC97. World J Gastroenterol. 2001;7(5):630-636.

34. Zhang W, Zhu XD, Sun HC, et al. Depletion of tumor-associated macrophages enhances the effect of sorafenib in metastatic liver cancer models by antimetastatic and antiangiogenic effects. Clin Cancer Res. 2010;16(13):3420-3430.

35. Zhao R, Wang TZ, Kong D, et al. Hepatoma cell line HepG2.2.15 demonstrates distinct biological features compared with parental HepG2. World J Gastroenterol. 2011;17(9):1152-1159.

36. Sun B-g, Zhang S-j, Liu Y-d, Yin L-r, Chen Z-x. Establishing human hepatoma model from subcutane to orthotopic transplantation with Bel-7402 cells in athymic mice and PTEN expression. Chinese Journal of Integrated Traditional And Western Medicine on Digestion. 2007;15(5):297-300.

37. Ding SJ, Li Y, Shao XX, et al. Proteome analysis of hepatocellular carcinoma cell strains, MHCC97-H and MHCC97-L, with different metastasis potentials. Proteomics. 2004;4(4):982-994.

38. Tang Z-Y, Sun F-X, Tian J, et al. Metastatic human hepatocellular carcinoma models in nude mice and cell line with metastatic potential. World J Gastroenterol. 2001;7(5):597-601.

39. Peng G, Hakim M, Broza YY, et al. Detection of lung, breast, colorectal, and prostate cancers from exhaled breath using a single array of nanosensors. British Journal of Cancer. 2010;103:542-551.

40. Zilberman Y, Ionescu R, Feng X, Müllen K, Haick H. Nanoarray of polycyclic aromatic hydrocarbons and carbon nanotubes for accurate and predictive detection in real-world environmental humidity. ACS Nano. 2011;5(8):6743-6753.

41. Zilberman Y, Tisch U, Shuster G, et al. Carbon nanotube/Hexa-perihexabenzocoronene bilayers for discrimination between nonpolar volatile organic compounds of cancer and humid atmospheres. $A d v$ Mater. 2010;22:4317-4320.

42. Zilberman Y, Tisch U, Pisula W, Feng X, Müllen K, Haick H. Sponge-like structures of Hexa-peri-hexabenzocoronenes derivatives enhances the sensitivity of chemiresistive carbon nanotubes to nonpolar volatile organic compounds. Langmuir. 2009;25(9):5411-5416.

43. Peng G, Trock E, Haick H. Detecting simulated patterns of lung cancer biomarkers by random network of single-walled carbon nanotubes coated with nonpolymeric organic materials. Nano Lett. 2008;8(11):3631-3635.

44. Konvalina G, Haick H. Effect of humidity on nanoparticle-based chemiresistors: a comparison between synthetic and real-world samples. ACS Appl Mater Interfaces. 2012;4(1):317-325.

45. Wilcoxon F. Individual comparisons by ranking methods. Biometrics Bulletin. 1945;1(6):80-83. 
46. Siegel S. Nonparametric statistics. The American Statistician. 1957; 11(3):13-19.

47. Ionescu R, Llobet E, Vilanova X, Brezmes J, Sueiras J, Calderer J. Quantitative analysis of $\mathrm{NO}_{2}$ in the presence of $\mathrm{CO}$ using a single tungsten oxide semiconductor sensor and dynamic signal processing. Analyst. 2002;127(9):1237-1246.

48. Tisch U, Billan S, Ilouze M, Phillips M, Peled N, Haick H. Volatile Organic Compounds in Exhaled Breath as Biomarkers for the Early Detection and Screening of Lung Cancer. CML Lung Cancer. 2012;5(4):107-117.

49. Fidler IJ. Critical factors in the biology of human cancer metastasis: twenty-eighth GHA. Clowes memorial award lecture. Cancer Res. 1990;19:6130-6138.

50. Rangarajan A, Weinberg RA. Opinion: Comparative biology of mouse versus human cells: modelling human cancer in mice. Nat Rev Cancer. 2003;3(12):952-959.

51. Anisimov VN, Ukraintseva SV, Yashin AI. Cancer in rodents: does it tell us about cancer in humans? Nat Rev Cancer. 2005;5(10):807-819.

52. Chen S, Zieve L, Mahadevan V. Mercaptans and dimethyl sulfide in the breath of patients with cirrhosis of the liver. Effect of feeding methionine. J Lab Clin Med. 1970;75(4):628-635.
53. Scislowski P, Pickard K. The regulation of transaminative flux of methionine in rat liver mitochondria. Archives of Biochemistry and Biophysics. 1994;314(2):412-416.

54. Ma S, Chan KW, Lee TKW, et al. Aldehyde dehydrogenase discriminates the CD133 liver cancer stem cell populations. Mol Cancer Res. 2008; 6:1146-1153.

55. Irwin R. NTP technical report on the toxicology and carcinogenesis studies ofbenzofuran (CAS no 271-289-6) in f344/n rats and b6c3f1 mice (gavage studies). Research Triangle Park, NC: US Department of Health and Human Services; 1989.

56. Fan H, Ren Y, Wu X, Wang Q. Synthesis and cytotoxicity of novel benzofuran neolignan derivatives. Journal of Chemical Research. 2010;34:233-235.

57. Manna SK, Bose JS, Gangan V, et al. Novel derivative of benzofuran induces cell death mostly by G2/M cell cycle arrest through p53dependent pathway but partially by inhibition of NF-kappaB. $J$ Biol Chem. 2010;285(29):22318-22327.

58. Ionescu R, Broza YY, Shaltieli H, et al. Detection of multiple sclerosis from exhaled breath using bilayers of Polycyclic Aromatic Hydrocarbons and single-wall carbon nanotubes. ACS Chem Neurosci. 2011;2:687-693. 


\section{Supplementary data}

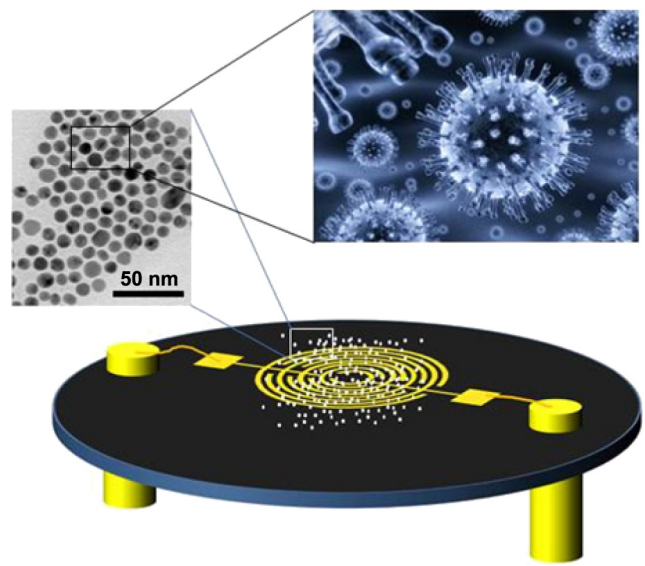

Figure SI Schematic representation of the GNP sensors used in this study (not drawn to scale).

Note: The left inset in the sensor's schematics shows a tunneling electron micrograph (TEM) of the GNPs in solution.

Abbreviations: GNP, gold nanoparticle; TEM, tunneling electron micrograph.

\section{Publish your work in this journal}

The International Journal of Nanomedicine is an international, peerreviewed journal focusing on the application of nanotechnology in diagnostics, therapeutics, and drug delivery systems throughout the biomedical field. This journal is indexed on PubMed Central, MedLine, CAS, SciSearch ${ }^{\circledR}$, Current Contents ${ }^{\circledR} /$ Clinical Medicine,
Journal Citation Reports/Science Edition, EMBase, Scopus and the Elsevier Bibliographic databases. The manuscript management system is completely online and includes a very quick and fair peer-review system, which is all easy to use. Visit http://www.dovepress.com/ testimonials.php to read real quotes from published authors. 